\title{
THE SPECTRUM OF THE COMPANION OF a SCORPII*
}

Seven spectrograms of the companion of $a$ Scorpii were obtained with the Cassegrain spectrograph of the McDonald Observatory on three nights of good seeing: April 20, July I, and July 6, I940. The dispersion of the instrument was $40 \mathrm{~A} / \mathrm{mm}$ at $\lambda 3933$, and the spectra extend to $\lambda$ 3200. The Balmer lines are broad and can be seen to about $H$ I6 or $H$ I 7 . This would place the $\mathrm{B} 4 \mathrm{n}$ star among the normal B stars of low luminosity in the diagram of Unsöld and Struve and does not reflect in the spectrum the well-established underluminous character of this object. ${ }^{2}$ The drop in intensity near the Balmer limit is conspicuous. There are no emission lines in the ultraviolet region, and the few absorption lines of $\mathrm{He}$ I are weak and diffuse.

The existence of moderately strong emission lines of $[\mathrm{Fe} \mathrm{II}]$, discovered by Wilson and Sanford ${ }^{3}$ in the ordinary photographic region, has been confirmed. The radial velocity, as determined from the two plates of best quality, is $-18.4 \mathrm{~km} / \mathrm{sec}$ for the absorption lines of $H$ and $H e \mathrm{I}$ and $+\mathrm{I} 2.5 \mathrm{~km} / \mathrm{sec}$ for the $[F e \mathrm{II}]$ emission lines. The difference between the absorption lines and the emission lines is doubtless real, but the large difference between our measures for the emission lines and those published by Wilson and Sanford, - O.I $\mathrm{km} / \mathrm{sec}$, may be due to a tendency in our observations to guide on one edge of the image of the companion. The slit of our spectrograph was set in position angle at right angles to the line joining the two stars, and it is possible that, in order to avoid the light of the bright star, we have illuminated the slit more on one side than on the other. The light of the red star is completely negligible to the violet side of $H \beta$. But in the visual region the overlapping is serious.

The principal purpose of these observations was an attempt to find whether the [Fe II] emission lines are present only in the light of the B-type companion, or whether they can be photographed in a region surrounding that star. On the first two exposures the star

* Contributions from the McDonald Observatory, University of Texas, No. 26.

I $A$ p. $J$., 91, 365, r940.

${ }^{2}$ Adams and Joy, Pub. A.S.P., 33, 206, 1921. $\quad 3$ Pub. A.S.P., 49, 221, r937. 
was permitted to drift along the slit (by means of the drive in declination, because the slit was oriented almost exactly north-south). An exposure of 45 minutes on Agfa Super-Pan-Press film produced a very strong spectrum, I.4 $\mathrm{mm}$ in width. If the emission lines originate in a nebulosity surrounding the star, this exposure-time should, therefore, be sufficient to bring out the lines when the star is held without motion on the slit. A spectrogram obtained in this manner gave a greatly overexposed continuous spectrum, which, because of photographic spreading, has a width of about 3.9 . The emission lines extend beyond the edges of the continuous spectrum and are sharp and strong. The extensions are about I".o in length, on each side of the continuous spectrum.

The evidence is, thus, strongly in favor of a small nebulosity surrounding the B-type companion. The extensions of the emission lines are so strong that they could hardly have been produced by an underexposed spectrum of scattered light from the B star or of light spilled over the normal tremor disk in moments of less satisfactory seeing. In order to test this, we obtained several short exposures, ranging from I minute to Io minutes, of the B-type companion, held stationary on the slit. On the shortest exposure the width of the continuous spectrum is I". 6 , and the density corresponds to the steepest part of the characteristic curve. Yet, in spite of a width greater than that of the extensions, the contrast between continuous spectrum and emission lines is so small that the latter are not visible with certainty. In fact, the contrast is so small that widened spectra are required to show them clearly.

There remains, of course, the possibility that the lines are variable in intensity. Not all of our observations were obtained on the same night, and a marked strengthening of the lines on July I with a decline in intensity on July 5 would also explain our results. Hence, it would be important to repeat the observations on several occasions.

If the nebulosity is confirmed, it would help in the interpretation of several other stars which, together with $a$ Scorpii, consist of a late-type star with a B-type companion and which show [Fe $\mathrm{\Pi}]$ emission lines. The normal appearance of the B spectrum of $a$ Scorpii and of other representatives of this group ${ }^{4}$ suggests that the emis-

${ }_{4}$ Ap. J., 91, 6r9, 1940. 
sion lines originate not in the reversing layers of these stars but in nebulosities whose presence is somehow connected with the existence of a cool supergiant in the system. The diameter of the nebulosity, $5 " .9$, appears reasonable in comparison to the separation of 3 " between the two components. In exceptionally good seeing it should be possible to set the slit between the two stars. But during the past season we have not been able to make such an observation.

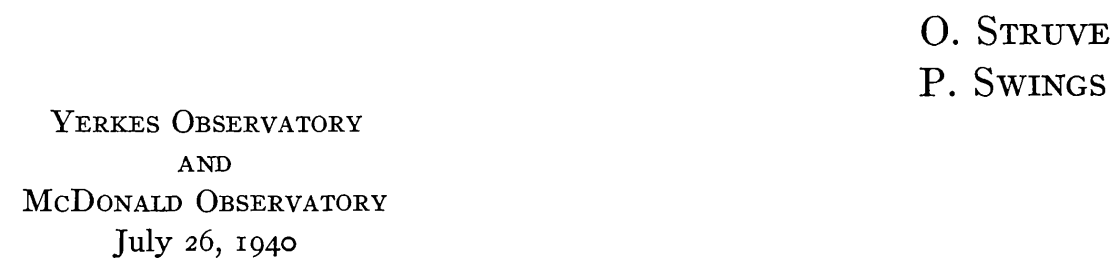

\section{ERRATUM}

In Volume 88 of this Journal:

On page 9I, middle of page: read " $\epsilon$ Capricorni (Pl. V)" instead of " $\epsilon$ Capricorni (Pl. IV)."

On page I05, eighth line from bottom: read "A close inspection of Plate II" instead of "A close inspection of Plate V." 